Journal of Computer Science 6 (6): 648-652, 2010

ISSN 1549-3636

(C) 2010 Science Publications

\title{
Psoriasis Detection Using Skin Color and Texture Features
}

\author{
${ }^{1}$ Nidhal K. El Abbadi, ${ }^{1}$ Nizar Saadi Dahir, ${ }^{2}$ Muhsin A. AL-Dhalimi and ${ }^{3}$ Hind Restom \\ ${ }^{1}$ Informatic Center for Research and Rehabilitation, \\ ${ }^{2}$ College of Medicine, \\ ${ }^{3}$ Department of Computer Science, College of Science, University of \\ Kufa, Najaf, Iraq
}

\begin{abstract}
Problem statement: In this study a skin disease diagnosis system was developed and tested. The system was used for diagnosis of psoriases skin disease. Approach: Present study relied on both skin color and texture features (features derives from the GLCM) to give a better and more efficient recognition accuracy of skin diseases. We used feed forward neural networks to classify input images to be psoriases infected or non psoriasis infected. Results: The system gave very encouraging results during the neural network training and generalization face. Conclusion: The aim of this worked to evaluate the ability of the proposed skin texture recognition algorithm to discriminate between healthy and infected skins and we took the psoriasis disease as example.
\end{abstract}

Key words: Skin recognition, skin texture, computer aided disease diagnosis, texture analysis, neural networks, psoriasis

\section{INTRODUCTION}

With advance of medical imaging technologies (including instrumentation, computer and algorithm), the acquired data information is getting so rich toward beyond the human's capability of visual recognition and efficient use for clinical assessment. The concept of computer or ideal interpreter has been around for many years. Computer technologies can make somewhat simple abnormality detection and disease diagnosis from the images, but could not replace the human ability to analyze complex information. On the other hand, computer technologies can "see" some details inside the images, while human might not be able to see. A computer interpreter can efficiently and consistently "read" many images, while human might make inconsistent assessments in an inefficient manner. Therefore, Computer Aided-Detection (CAD) and Computer Aided Diagnosis (CADx) become more desirable and are now under development by many research groups in the world.

Computer aided disease diagnosis proven to be very helpful and many systems where used to help in the diagnosis of many diseases:

- Lung cancer-currently, radiologist can fail to detect lung nodules in up to $30 \%$ of actually positive cases. If a computerized scheme could alert the radiologist to locations of suspected nodules, then potentially the number of missed nodules could be reduced. Furthermore the evaluation of diffuse interstitial disease is one of the most difficult problems in diagnostic radiology. A thoracic CT scan generates about 240 section images for radiologists to interpret (Acharya and Ray, 2005)

- Chest radiography-computerized automated analysis of heart sizes; an automated method is being developed for determining a number of parameters related to the size and shape of the heart and of the lung in chest radiographs (60 chest radiographs were generally acceptable to radiologist for the estimation of the size and area of the heart project)

- Colon cancer-colon cancer is the second leading case of cancer deaths for men and woman in the USA. Most colon cancers can be prevented if precursor colonic polyps are detected and removed. CT colonography (virtual colonoscopy) is being examined as a potential screening device (400-700 images) (Acharya and Ray, 2005)

- Verma and Zakos (2001) presented a based on fuzzy-neural and feature extraction techniques for detecting and diagnosing micro calcifications' patterns. A in digital mammograms fuzzy technique in conjunction with three features: Entropy, standard deviation and number of pixels, was used to detect a micro calcification pattern.

Corresponding Author: Nidhal K. El Abbadi, Informatic Center for Research and Rehabilitation, University of Kufa, Najaf, Iraq 
Then, a neural network was used to classify it into benign or malignant

- Computer aided diagnosis of bone lesions in the jaw, based on image analysis and content-based query of a database. A lesion in a digitized radiograph of the jaw is marked and then outlined by a region-based active contour model. Then gray level, texture and other features are computed for the suspicious region

- Developed a new tool to assess retinopathy of prematurity. This technique captures the image of the retina to extract and quantify both tortuosity and dilation of blood vessels

Artificial Neural Network Computer-Aided Diagnosis (ANNCAD) scheme for the diagnosis of Alzheimer's disease with multi-center data using the registration and region based statistics.

In this study we developed a new algorithm that can be used to detect the deformation in abnormal skin's texture and color due to infection and to recognize skins that have a specific disease form those with other diseases. The disease we chosen is psoriasis.

Many skin recognition and modeling methods depends on skin color (Hwei-Jen et al., 2005) which have many difficulties. The skin color depends on human race and on lighting conditions, although this can be avoided in some ways using $\mathrm{YCbCr}$ color spaces in which the two components $\mathrm{Cb}$ and $\mathrm{Cr}$ depend only on chrominance, there still many problems with this method because there are many objects in the real world that have a chrominance in the range of the human skin which may be wrongly considered as skin. Also many skin diseases will cause deformation in skin's texture as well as the color. For the above reasons combining the texture features of skin with its color feature will increase the accuracy of skin disease diagnosis systems.

Psoriasis: Psoriasis is a common, chronic, relapsing, inflammatory skin disorder with a strong genetic basis (Giardina et al., 2004). One to $2 \%$ of the American population has plaque psoriasis. The plaque type is the most common, although several other distinctive clinical variants of psoriasis are recognized (e.g., Psoriasis, Guttate; Psoriasis, Nails; Psoriasis, Pustular) (Naldi, 2004). Plaque psoriasis is most typically characterized by circular-to-oval red plaques distributed over extensor body surfaces and the scalp. The plaques usually exhibit scaling as a result of epidermal hyper proliferation and dermal inflammation (Naldi and Rzany, 2005). The extent and duration of the disease is highly variable from patient to patient. Acute flares or relapses of plaque psoriasis may also evolve into more severe disease, such as pustular or erythrodermic psoriasis. The diagnosis of psoriasis is usually apparent clinically but sometimes it nay resembles other skin disorders in atypical cases, such as lichen simplex chronicus, nummular eczema, seborrheic dermatitis and tinea corporis.

Related work: Number of comparative studies of skin color pixel classification has been reported. (Jones and Rehg, 1999) created the first large skin database-the Compaq database-and used the Bayesian classifier with the histogram technique for skin detection. Brand and Mason (2000) compared three different techniques on the Compaq database: thresholding the red/green ratio, color space mapping with $1 \mathrm{D}$ indicator and RGB skin probability map. Terrillon et al. (2000) compared Gaussian and Gaussian mixture models across nine chrominance spaces on a set of 110 images of 30 Asian and Caucasian people. Shin et al. (2000) compared skin segmentation in eight color spaces. In their study, skin samples were taken from the AR and the University of Oulo face databases and non-skin samples were taken from the University of Washington image database. Al Abbadi et al. (2008) used skin texture and color features to recognizing skin texture from non skin texture.

\section{Feature extraction:}

Color features (color moment): Color moment is a compact representation of the color feature to characterize a color image. It has been shown that most of the color distribution information is captured by the three low-order moments. The first-order moment $\left(\mu_{c}\right)$ captures the mean color, the second-order moment $\left(\sigma_{c}\right)$ captures the standard deviation and the third-order moment captures the skewness $\left(\theta_{c}\right)$ of color. These three low-order moments $\left(\mu_{\mathrm{c}}, \sigma_{\mathrm{c}}, \theta_{\mathrm{c}}\right)$ are extracted for each of the three color planes ( $R$ G B), using the following mathematical formulation:

$$
\begin{aligned}
& \mu_{c}=\frac{1}{M N} \sum_{i=1}^{M} \sum_{j=1}^{N} p_{i j}^{c} \\
& \sigma_{c}=\left[\frac{1}{M N} \sum_{i=1}^{M} \sum_{j=1}^{N}\left(p_{i j}^{c}-\mu_{c}\right)^{2}\right]^{1 / 2} \\
& \theta_{c}=\left[\frac{1}{M N} \sum_{i=1}^{M} \sum_{j=1}^{N}\left(p_{i j}^{c}-\mu_{c}\right)^{3}\right]^{1 / 3}
\end{aligned}
$$


where, $p^{c_{i}}$ is value of the $c^{\text {th }}$ color component of the color pixel in the $\mathrm{i}^{\text {th }}$ row and $\mathrm{j}^{\text {th }}$ column of the image. As a result, we need to extract only nine parameters (three moments for each of the three color planes) to characterize the color.

Texture features: Texture is a very interesting image feature that has been used for characterization of images, a major characteristic of texture is the repetition of a pattern or patterns over a region in an image. The elements of patterns are sometimes called textons. The size, shape, color and orientation of the textons can vary over the region. The difference between two textures can be in the degree of variation of the textons. It can also be due to spatial statistical distribution of the textons in the image. Texture is an innate property of virtually all surfaces, such as bricks, fabrics, woods, papers, carpets, clouds, trees, lands and skin. It contains important information regarding underlying structural arrangement of the surfaces in an image.

Texture analysis has been an active area of research in pattern recognition. A variety of techniques have been used for measuring textural similarity. proposed co-occurrence matrix representation of texture features to mathematically represent gray level spatial dependence of texture in an image. In this method the co-occurrence matrix is constructed based on the orientation and distance between image pixels. Meaningful statistics are extracted from this cooccurrence matrix, as the representation of texture. Since basic texture patterns are governed by periodic occurrence of certain gray levels, co-occurrence of gray levels at predefined relative positions can be a reasonable measure of the presence of texture and periodicity of the patterns. Several texture features such as entropy, energy, contrast and homogeneity, can be extracted from the co-occurrence matrix of gray levels of an image.

The gray level co-occurrence matrix $\mathrm{C}(\mathrm{i}, \mathrm{j})$ is defined by first specifying a displacement vector $\mathrm{d}_{\mathrm{xy}}=$ $\left(\delta_{\mathrm{x}}, \delta_{\mathrm{y}}\right)$ and then counting all pairs of pixels separated by displacement $d_{x y}$ and having gray levels $i$ and $j$. The matrix $C(i, j)$ is normalized by dividing each element in the matrix by the total number of pixel pairs. Using this co-occurrence matrix, the texture features metrics are computed as follows:

Entropy $=\sum_{\mathrm{i}} \sum_{\mathrm{j}} \mathrm{C}(\mathrm{i}, \mathrm{j}) \log (\mathrm{C}(\mathrm{i}, \mathrm{j})$

Energy $=\sum_{i} \sum_{j} C^{2}(i, j)$
Contrast $=\sum_{\mathrm{i}} \sum_{\mathrm{j}}(\mathrm{i}-\mathrm{j})^{2} \mathrm{C}(\mathrm{i}, \mathrm{j})$

Homogeneity $=\sum_{i} \sum_{j} \frac{C(i, j)}{1+|i-j|}$

These four features are combined with the nine features computed for each color component resulting in 13 element features vector use to characterize and model the skin in this study.

The proposed skin disease diagnosis algorithm: Our proposed skin disease diagnosis algorithm consists of three main tasks as shown in Fig. 1:

- Creation of the library of representative skin features

- Neural network training

- Classification

Skin library: The library of skin texture is comprised of 24 images of skin images of size $300 \times 300$ pixel. The library consisted of variety of skin types of different human races, different places of the human body and different lighting conditions, with 12 samples that have psoriasis and 12 that have other different skin diseases. Samples of that library are shown in Fig. 2.

The structure of the neural network: We used a feed forward feed-forward back propagation Neural Network (NN)) with adaptable learning rate. The NN have 3 layer; an input layer (13 neuron), a hidden layer (50 neuron) and output layer (1 neuron). The activation function used is the tan sigmoid function, for both the hidden and the output layer. The input to the neural network is the feature vector containing 13 component these are the 4 texture feature and the three color moments for each color component (R G B), the NN has only one output as shown in Fig. 3.

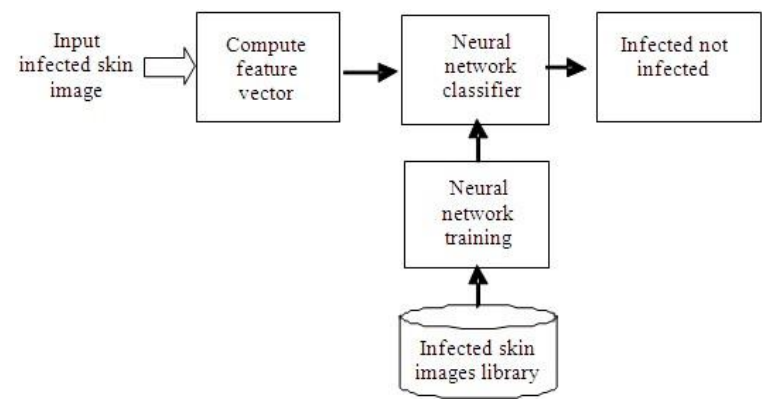

Fig. 1: Skin disease diagnosis algorithm tasks 650 

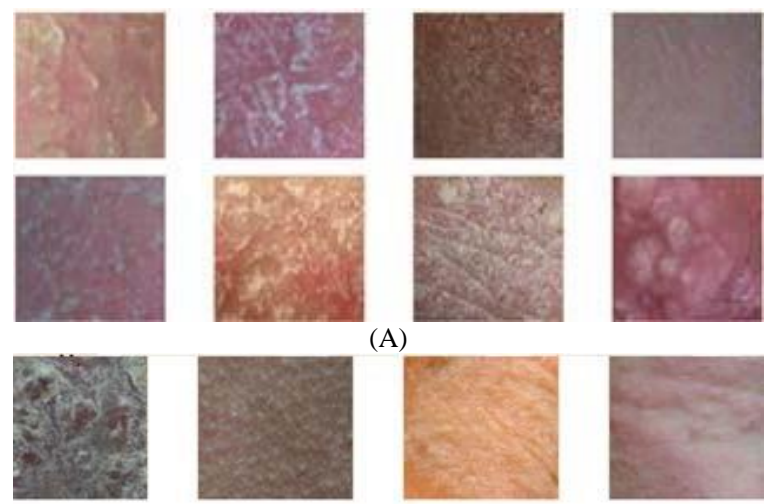

(A)
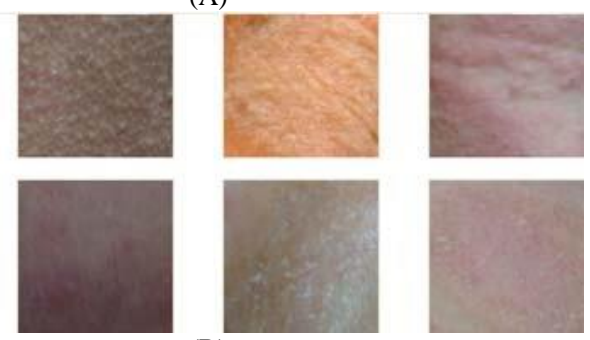

(B)

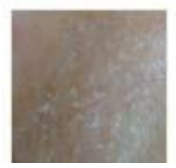

Fig. 2: The skin library samples (A) Samples with psoriasis (B) Samples with other diseases

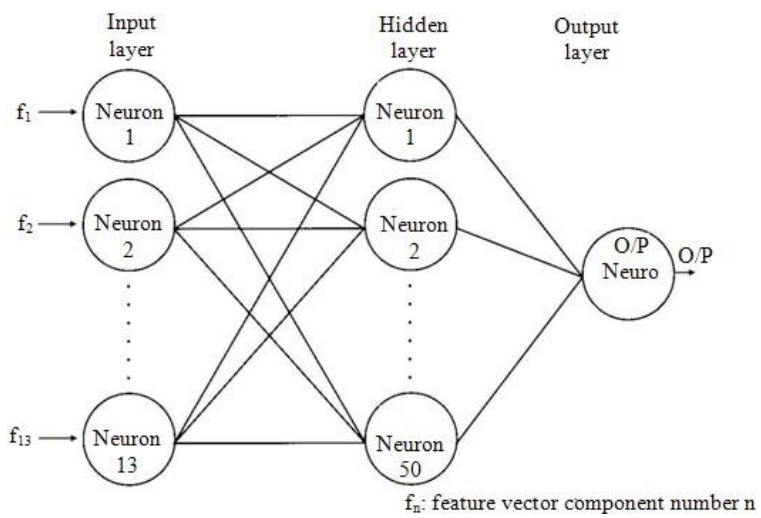

Fig. 3: The neural network structure

\section{MATERIALS AND METHODS}

The images of the skin library where taken by 10 Mega Pixel digital camera in a dermatology clinic over a 3 months period and was classified and indexed to be suitable for our system. The algorithm was simulated using Matlab ${ }^{\circledR} 7.0$.

\section{RESULTS}

The NN training process is done using skin images with psoriasis and skin images with other diseases from the image library. The output of the neural network assumed to be 1 for psoriasis infected skin image and 1 for input images for skins with other diseases.

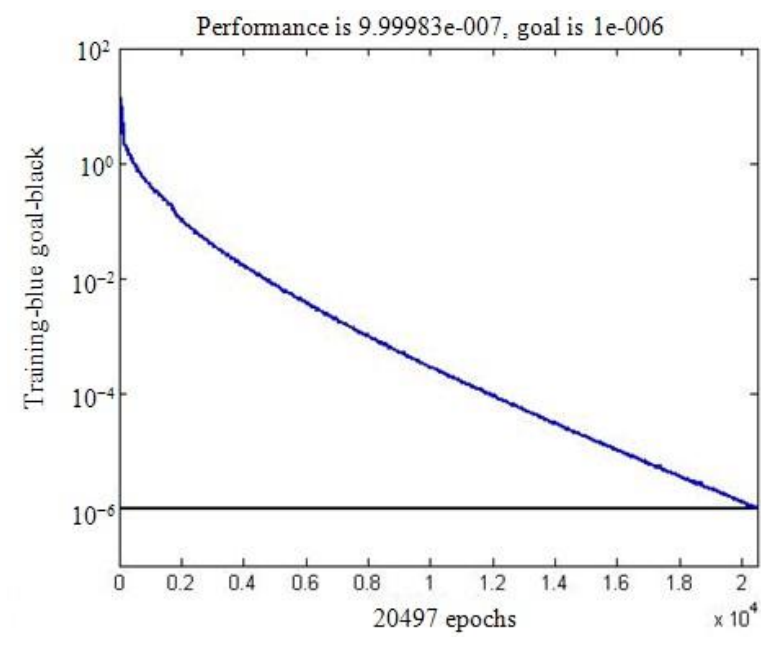

Fig. 4: Neural network performance

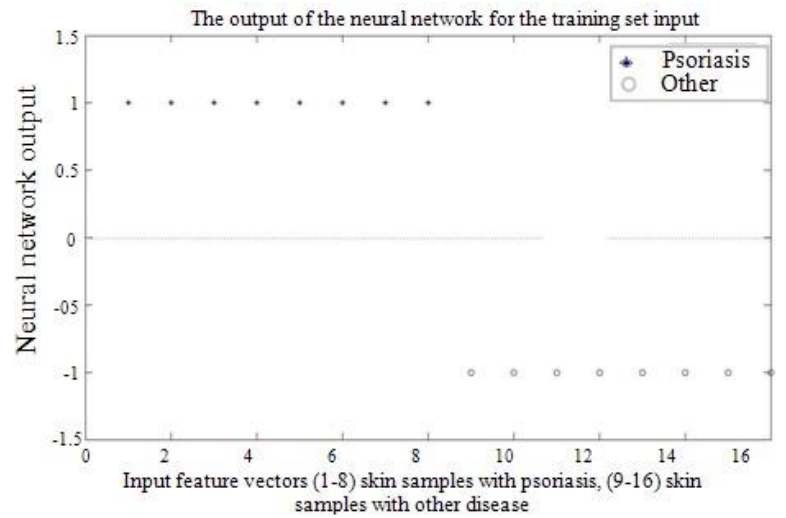

Fig. 5: Neural network $\mathrm{O} / \mathrm{P}$ for the training set input images

The performance criterion used is Sum Square Error (SSE) and the goal was $10^{-6}$ which is a very proved to be very acceptable goal. The performance goal is reached after 20497 training iteration (Fig. 4).

To test our system we used (4) images infected with psoriasis and 4 images infected with other diseases. These images were not used in the training phase. The images were input to the neural network after training to obtain the generalization results as shown in Fig. 5 and 6.

Figure 5 shows the output the neural network for the training set. Figure 6 shows the output of the same neural network for a testing set of 8 samples (4 with psoriasis and 4 with other disease). The desired output is (1) for psoriasis infected images and (-1) for non psoriasis infected images. 


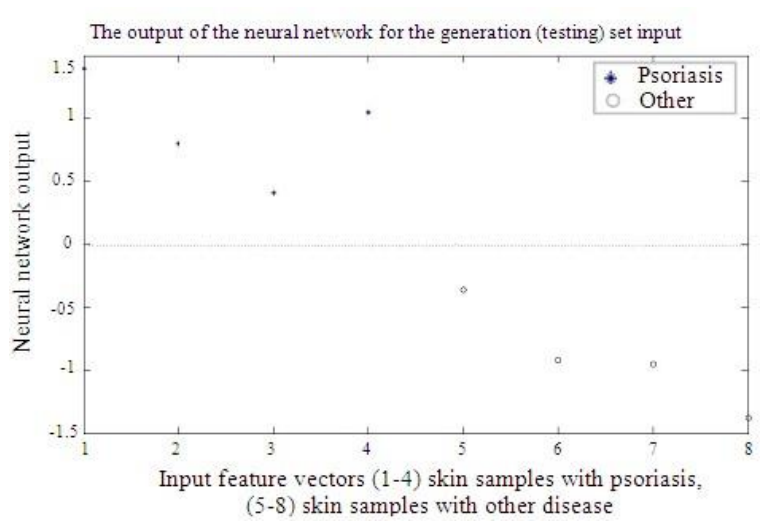

Fig. 6: Neural network generalization O/P for non-skin input images

\section{DISCUSSION}

It can be seen from Fig. 6 that although the outputs are deviated form the desired output, they still can be recognized correctly since they did not exceed the threshold which is 0 in this case. Hence all the samples can be correctly classified.

\section{CONCLUSION}

The aim of this works it to evaluate the ability of the proposed skin texture recognition algorithm to discriminate between healthy and infected skins and we took the psoriasis disease as example.

It can be concluded from Fig. 5 and 6 that the system gave very encouraging results. The used texture and color features enhanced the performance of our system and gave high recognition accuracy as shown in Fig. 6. This accuracy proves that the texture features are very useful as recognition features for diagnosis of the psoriasis.

The system can be also used for detecting other skin diseases by choosing the proper training sets.

\section{REFERENCES}

Acharya, T. and J.K. Ray, 2005. Image Processing Principles and Applications. Wiley-Interscience, ISBN: 10: 0471719986, pp: 428.

Al Abbadi, N.K., N.S. Dahir and Z.A. Alkareem, 2008. Skin texture recognition using neural networks. Proceeding of the Arab Conference for Information Technology, (ACIT’08), Hammamat, Tunisia, pp: $1-4$.

http://eref.uqu.edu.sa/files/eref2/folder6/f39.pdf
Brand, J. and J.S. Mason, 2000. A comparative assessment of three approaches to pixel-level human skin detection. Proc. Int. Conf. Patt. Recog., 1: 1056-1059. DOI: 10.1109/ICPR.2000.905653

Giardina, E., C. Sinibaldi and G. Novelli, 2004. The psoriasis genetics as a model of complex disease. Curr. Drug Targets-Inflamm. Allergy, 3: 129-136. DOI: 10.2174/1568010043343877

Hwei-Jen, L., W. Shu-Yi, Shwu-Huey and Y. Ta-Kao, 2005. Face detection based on skin color segmentation and neural network. IEEE Trans. Control Syst. Technol., 2: 1144-1149. DOI: 10.1109/ICNNB.2005.1614818

Jones, M.J. and J.M. Rehg, 1999. Statistical color models with application to skin detection. Proceedings of the 1999 IEEE Computer Society Conference on Computer Vision and Pattern Recognition, June 23-25, IEEE Computer Society, Fort Collins, CO., USA., pp: 274, DOI: 10.1109/CVPR.1999.786951

Naldi, L. and B. Rzany, 2005. Traditional therapies in the management of moderate to severe chronic plaque psoriasis: An assessment of the benefits and risks. Br. J. Dermatol., 152: 597-615. DOI: 10.1111/J.1365-2133.2005.06563.X

Naldi, L., 2004. Epidemiology of psoriasis. TargetsInflamm. Allergy J., 3: 121-128. DOI: 10.2174/1568010043343958

Shin, M.C., K.I. Chang and L.V. Tsap, 2002. Does colorspace transformation make any difference on skin detection? Proceeding of the 6th IEEE Workshop Applications of Computer Vision, Dec. 3-4, IEEE Computer Society, Washington DC., USA., pp: 275-279. http://portal.acm.org/citation.cfm?id=836826

Terrillon, J.C., H. Fukamachi, S. Akamatsu and M.N. Shirazi, 2000. Comparative performance of different skin chrominance models and chrominance spaces for the automatic detection of human faces in color images. Proceedings of the 4th IEEE International Conference on Automatic Face and Gesture Recognition, Mar. 26-30, IEEE Computer Society, Washington DC., USA., pp: 5461. DOI: 10.1109/AFGR.2000.840612

Verma, B. and J. Zakos, 2001. A computer-aided diagnosis system for digital mammograms based on fuzzy-neural and feature extraction techniques. IEEE Trans. Inform. Technol. Biomed., 5: 46-54. PMID: 11300216 